\title{
Bacteriome and mycobiome and bacteriome-mycobiome interactions in head and neck squamous cell carcinoma
}

\author{
Elizabeth Shay ${ }^{1,2}$, Naseer Sangwan ${ }^{3}$, Roshan Padmanabhan ${ }^{1}$, Scott Lundy ${ }^{1,4}$, Brian \\ Burkey ${ }^{5}$ and Charis Eng ${ }^{1,5,6}$ \\ ${ }^{1}$ Genomic Medicine Institute, Lerner Research Institute, Cleveland Clinic, Cleveland, Ohio, USA \\ ${ }^{2}$ Cleveland Clinic Lerner College of Medicine, Case Western Reserve University School of Medicine, Cleveland, Ohio, USA \\ ${ }^{3}$ Center for Microbiome and Human Health, Lerner Research Institute, Cleveland Clinic, Cleveland, Ohio, USA \\ ${ }^{4}$ Glickman Urological and Kidney Institute, Cleveland Clinic, Cleveland, Ohio, USA \\ ${ }^{5}$ Taussig Cancer Institute, Cleveland Clinic, Cleveland, Ohio, USA \\ ${ }^{6}$ Department of Genetics and Genome Sciences, Case Western Reserve University School of Medicine, Cleveland, Ohio, USA \\ Correspondence to: Charis Eng, email: engc@ccf.org \\ Keywords: microbiome; bacteriome; mycobiome; head and neck squamous cell carcinoma; cancer
} Received: March 17, $2020 \quad$ Accepted: May 14, $2020 \quad$ Published: June 23, 2020

Copyright: Shay et al. This is an open-access article distributed under the terms of the Creative Commons Attribution License 3.0 (CC BY 3.0), which permits unrestricted use, distribution, and reproduction in any medium, provided the original author and source are credited.

\section{ABSTRACT}

The etiology of head and neck squamous cell carcinoma (HNSCC) is not fully understood. While risk factors such as positive human papilloma virus (HPV) status, smoking and tobacco use have been identified, they do not account for all cases of the disease. We aimed to characterize the bacteriome, mycobiome and mycobiomebacteriome interactions of oral wash in HNSCC patients and to determine if they are distinct from those of the oral wash of matched non-HNSCC patients. Oral wash samples were collected from 46 individuals with HNSCC and 46 controls for microbiome analyses. We identified three fungal phyla and eleven bacterial phyla of which Ascomycota (fungi, $72 \%$ ) and Firmicutes (bacteria, 39\%) were the most dominant, respectively. A number of organisms were identified as being differentially abundant between oral wash samples from patients with HNSCC and oral wash samples from those without HNSCC. Of note, strains of Candida albicans and Rothia mucilaginosa were differentially abundant and Schizophyllum commune was depleted in those with HNSCC compared to oral wash from those without HNSCC. Our results suggest that the oral cavity of HNSCC patients harbors unique differences in the mycobiome, bacteriome, and microbiome interactions when compared to those of control patients.

\section{INTRODUCTION}

Head and neck squamous cell carcinoma (HNSCC) refers to cancer arising from the squamous epithelium of the oral cavity, pharynx, nasopharynx, and larynx. This constellation of diseases results in greater than 300,000 deaths annually, and those that do survive often suffer from significantly impaired quality of life [1,2]. Cigarette smoking, tobacco use, and positive human papillomavirus (HPV) status are well-known risk factors for HNSCC [3]. Approximately, three-quarters of HNSCC cases can be attributed to cigarette smoking and tobacco use $[4,5]$. The percentage of HNSCC attributable to HPV continues to increase and has been estimated to be as high as $39.8 \%$ at some sites [6-8]. Betel quid chewing, which is popular in India and other Asian countries, has also been shown to increase the risk of HNSCC (mainly oral cancer) [9]. Occupational exposure to substances such as wood dust, coal dust, welding fumes, asbestos and formaldehyde has been reported to promote the development of HNSCC [10]. HNSCC can also be the result of germline mutations, with Fanconi anemia being the most well-known example 
$[11,12]$. Patients with Fanconi anemia, a heritable syndrome characterized by genomic instability, develop HNSCC at higher incidences and younger ages than the general population [13].

However, not all patients with these risk factors develop HNSCC, and some patients with HSNCC lack these risk factors. Importantly, the diagnosis of HNSCC is often delayed due to non-specific symptoms and lack of an established screening tool [14]. There is, therefore, a need to identify additional risk factors to better predict which patients, particularly among those at high risk, will develop HNSCC.

The microbiome is one important factor that may play a role in carcinogenesis, with the relationship between Helicobacter pylori and gastric adenocarcinoma serving as a well-established example [15]. The contribution of the microbiome to HNSCC pathogenesis, however, has not been fully explored. Dysbiosis, or alterations in the composition of microbial communities, has previously been implicated in periodontal disease [16, 17]. This is noteworthy, as the association between chronic periodontitis and HNSCC thus implies a role for dysbiosis in these cancers $[18,19]$. More direct associations between HNSCC and dysbiosis have also been found. Our pilot study found evidence of epigenetic changes in HNSCC genes that were associated with certain microbial subpopulations [20]. We extended this work by demonstrating the relative depletion of certain bacterial communities in paired tumor (HNSCC) versus normal oral epithelium samples, a finding that was correlated with the extent of disease [21]. These findings highlight the association of oral dysbiosis with HNSCC.

The oral microbiome contains not only bacterial communities (bacteriome) but also fungal communities comprising the oral mycobiome [22]. Fungal communities have the potential not only to independently influence the environment of the oral cavity, but also to interact with oral bacterial communities. Recently, our group found differences in bacteriome and mycobiome correlations in oral tongue cancer (a type of HNSCC not commonly associated with HPV) compared to normal oral epithelial tissue [23]. Bacteriome-mycobiome correlations (i. e., cross-talk between the communities that is biologically relevant) from oral wash specimens have been less well characterized. Compared to that of tissue biopsies, the procedure to obtain oral wash specimens is rapid, inexpensive, and non-invasive. Determining bacteriome and mycobiome profiles as well as their correlations within oral wash samples could facilitate the development of a potential screening and high-risk surveillance tool.

We, therefore, sought to identify and characterize differences in the bacteriome and mycobiome profiles of patients with HNSCC versus healthy cancer-free patients, using oral wash as template biospecimen. To accomplish this, we performed 16S rRNA and ITS gene sequencing on oral washes from HNSCC patients and matched healthy individuals, followed by bioinformatics analysis.

\section{RESULTS}

\section{Participant characteristics}

We used available oral wash DNA from 46 HNSCC participants and 46 matched control participants in this study (Table 1).

\section{Composition of the bacteriome and mycobiome of oral wash}

Of the 92 samples sent for sequencing, 79 had a sufficient number of reads for mycobiome analysis. We identified three fungal phyla in the oral wash samples. Ascomycota was the predominant phylum (72.0\%), followed by Basidiomycota (27.3\%) and Mucoromycota $(0.7 \%)$.

Of the DNA from the 92 original oral wash samples sent for sequencing, 85 had sufficient reads for bacteriome analysis. Eleven bacterial phyla were identified. Firmicutes was the most dominant (39.2\%), followed by Bacteroidetes (23.2\%), Actinobacteria (15.4\%), Proteobacteria (9.7\%), and Fusobacteria $(8.0 \%)$. Other phyla present in marginal amounts were Spirochaetes (1.8\%), Saccharibacteria (TM7) $(1.8 \%)$, Synergistetes $(0.6 \%)$, Absconditabacteria (SR1) $(0.3 \%)$, Gracilibacteria $(0.03 \%)$ and Chloroflexi $(0.01 \%)$.

Taxonomic composition (genus level) for both mycobiome and bacteriome stratified by disease status is summarized below (Figure 1).

\section{Diversity of the oral bacteriome and mycobiome of HNSCC versus control patients}

In order to determine if within-sample diversity ( $\alpha$-diversity) of each group differed, we calculated the Shannon diversity index (which takes into account both evenness and richness of communities) of the samples in each group. Evaluation of the bacteriome revealed that the $\alpha$-diversity of case oral wash, as measured by the Shannon diversity index, was reduced relative to control oral wash (Shannon $p<0.05$, Figure 2A). When evaluating the mycobiome, the $\alpha$-diversity (Shannon diversity index) of HNSCC oral wash was noted to be reduced relative to that of control oral wash ( $p<0.05$, Figure 2B). Comparison of $\alpha$-diversity by site demonstrated statistically significant differences between sub-groups (Supplementary Figure 1).

Bray-Curtis dissimilarity index was used to determine differences in the taxonomic composition (bacterial) between the case and control groups ( $\beta$-diversity) (Figure $2 \mathrm{C}$ ). Oral wash samples clustered similarly and there was no statistically significant difference between the groups $(p=0.054)$. Similar analysis was undertaken to compare how cancer and control groups differed based on fungal taxonomic 
Table 1: Participant characteristics

\begin{tabular}{|c|c|c|}
\hline & Cancer (46) & Normal (46) \\
\hline Age (years) & $60 \pm 13$ & $59 \pm 12$ \\
\hline Male & $31(67.4 \%)$ & $31(67.4 \%)$ \\
\hline \multicolumn{3}{|l|}{ Race } \\
\hline White & $44(95.7 \%)$ & $44(95.7 \%)$ \\
\hline Black & $2(4.4 \%)$ & $2(4.4 \%)$ \\
\hline \multicolumn{3}{|l|}{ Overall Stage } \\
\hline Stage I-II & $17(37.0 \%)$ & \\
\hline Stage III-IV & $27(58.7 \%)$ & \\
\hline Unknown & $2(4.4 \%)$ & \\
\hline \multicolumn{3}{|l|}{ Smoking History } \\
\hline Current & $3(6.6 \%)$ & $1(2.2 \%)$ \\
\hline Never & $21(45.7 \%)$ & $41(89.1)$ \\
\hline Past & $22(47.8 \%)$ & $4(8.7 \%)$ \\
\hline \multicolumn{3}{|l|}{ Alcohol Use } \\
\hline Yes & $27(41.3)$ & $34(73.9 \%)$ \\
\hline No & $19(58.7 \%)$ & $10(21.7 \%)$ \\
\hline Unknown & $0(0 \%)$ & $2(4.3 \%)$ \\
\hline \multicolumn{3}{|l|}{ Site } \\
\hline Floor of Mouth (FOM) & $2(4.4 \%)$ & \\
\hline Larynx & $7(15.2 \%)$ & \\
\hline Oral cavity & $3(6.5 \%)$ & \\
\hline Other & $4(8.7 \%)$ & \\
\hline Pharynx/Hypopharynx & $1(2.17 \%)$ & \\
\hline Tongue & $22(47.8 \%)$ & \\
\hline Tonsil & $7(15.2 \%)$ & \\
\hline \multicolumn{3}{|l|}{ HPV Status } \\
\hline Positive & $11(23.9 \%)$ & \\
\hline Negative & $1(2.2 \%)$ & \\
\hline Missing & $34(74.0 \%)$ & \\
\hline
\end{tabular}

composition (Figure 2D). Oral wash samples in both cohorts clustered separately by disease status $(p=0.01)$. $\beta$-diversity comparisons of the groups by site of cancer showed that samples clustered separately by site for both the mycobiome and bacteriome (Supplementary Figure 1). Samples also clustered separately when analyzing ethanol use and smoking history. (Supplementary Figure 2).

\section{Differential abundance analysis}

Analysis (taking into account smoking and ethanol use history) was conducted to determine which organisms were differentially abundant when comparing oral wash obtained from case versus control participants (Figure 3 ). Specific strains of Candida albicans were found to be both overrepresented and underrepresented in oral wash samples from cancer patients relative to those from non-cancer controls (Figure 3A). Candida dubliniensis, Schizophyllum commune and an organism from the class Agaricomycetes were found to be overrepresented in controls relative to cases. By contrast, one strain of Neoascochyta exitialis was found to be underrepresented in oral wash from cases relative to that of controls.

Differential abundance analysis of bacteria in oral wash revealed two strains of Rothia mucilaginosa that were overrepresented in the oral wash of cases relative to that of controls (Figure 3B). Additionally, one strain of Rothia mucilaginosa was underrepresented in case oral wash versus control oral wash. Strains of Streptococcus parasanguinis, Gemella sanguinis and an organism from the genera Veillonella were overrepresented in case oral wash versus control oral wash. Strains of Haemophilus parainfluenzae, Streptococcus cristatus, Veillonella 
rogosae and an organism from the genera Fusobacterium were underrepresented in cancer oral wash relative to control oral wash.

Differential abundance analysis comparing ethanol use and smoking history was performed for the bacteriome and mycobiome (Figure 4A-4D).

\section{Exploring microbe-microbe interactions with network analysis}

Network analysis revealed the presence of intra-and inter kingdom interactions within the oral wash of case and control participants (Figure 5). Each network comprised of multiple clusters containing predominantly bacterial organisms and fewer fungal organisms. The two largest clusters in both case and control networks were connected and contained many of the same organisms. Interestingly, the interaction between Peptostreptococcaceae and Saccharibacteria was negative in case oral wash but positive in control oral wash. Similarly, Prevotella and Freitibacterium correlated negatively in case oral wash, but positively in control oral wash. Additionally, Candida

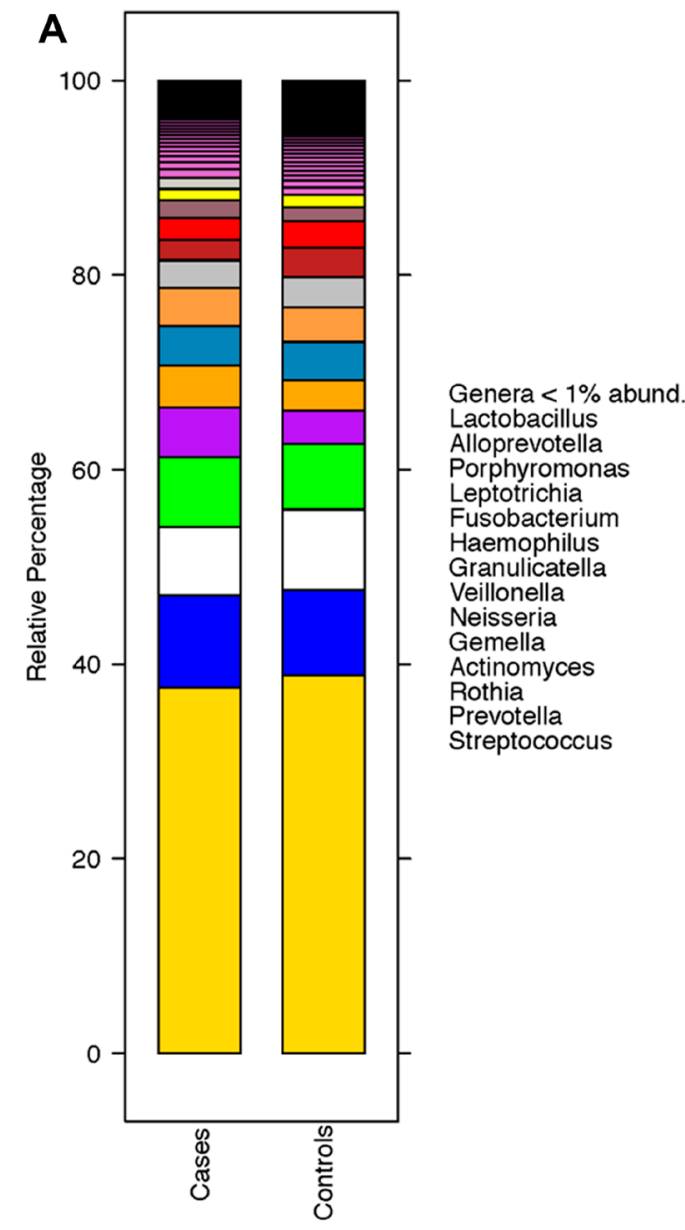

correlated negatively with Alloscardovia in case oral wash, but positively in control oral wash.

\section{DISCUSSION}

Previous studies have shown associations between the oral microbiome and HNSCC. These studies, have, however, primarily focused on the bacteriome. This study is one of a few that have explored the mycobiome profiles in HNSCC from oral wash [23-25]. Here, we show that individual bacterial and fungal organisms display association with HNSCC status and that these microorganisms interact with each other.

The oral mycobiomes of both HNSCC and healthy participants were dominated by the genus Candida (Figure 1). This observation has been reported in several studies of the oral mycobiome in healthy and diseased states $[22,26,27]$. We also observed that varying strains of Candida albicans were enriched in both diseased and healthy participants. Oral candidiasis has been strongly associated with the risk of development of various malignancies, including those of the head and neck

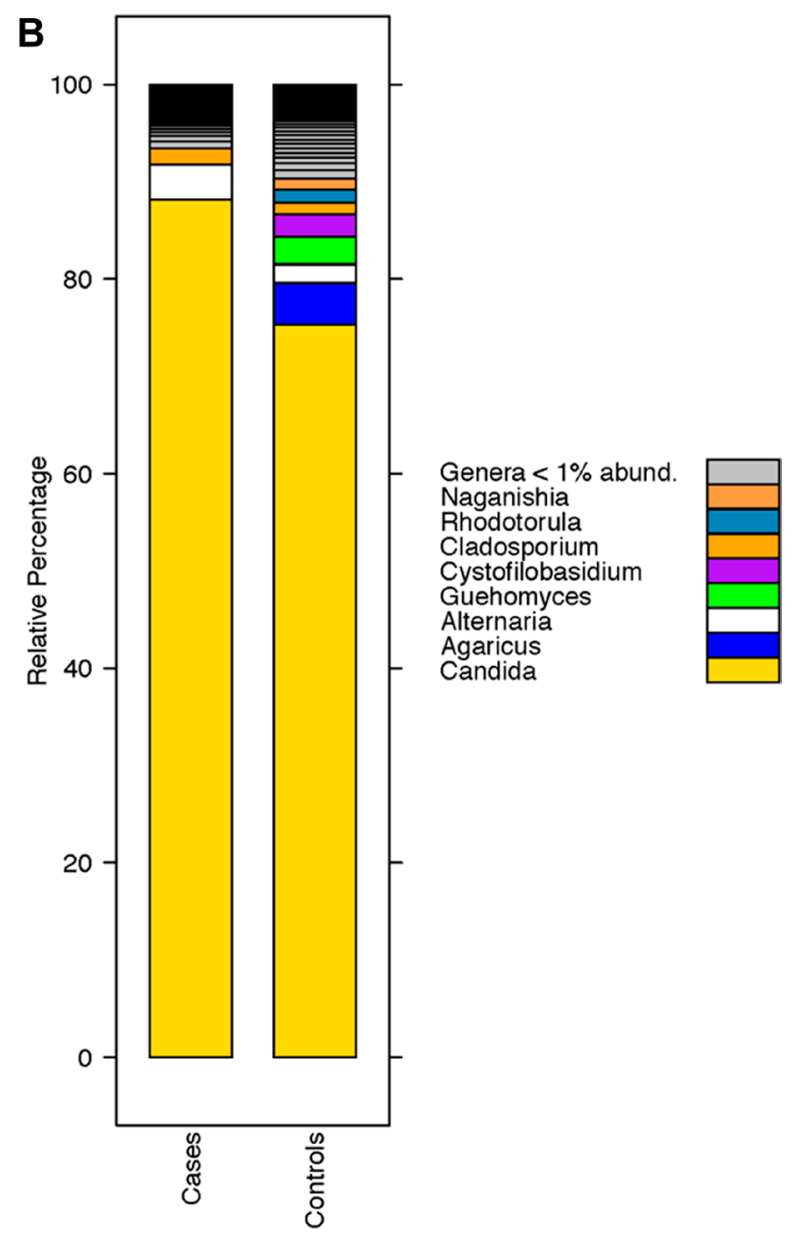

Figure 1: Taxonomic composition of the oral wash microbiome of head and neck squamous cell carcinoma (HNSCC) at the genus level. (A) Composition of the oral wash bacteriome (genus level) of cases (HNSCC patients) versus controls. (B) Composition of the oral wash mycobiome (genus level) of cases versus controls. 
[28]. Perera et al. similarly noted that Candida albicans was elevated in oral squamous cell carcinoma tissue compared to benign tissue (intra-oral fibro-epithelial polyps) [24]. Vesty et al. also reported an abundance of Candida albicans in the saliva of HNSCC patients that correlated with the abundance of the inflammatory cytokines IL-1 $\beta$ and IL-8 [25]. This finding implies a role for Candida albicans in inducing inflammation, perhaps via hypermethylation of tumor suppressors [25, 29]. That we observed enrichment of $C$. albicans in both healthy and diseased participants does not negate evidence that the organism may be involved in HNSCC carcinogenesis. It is known, for example, that the association of H. Pylori with gastric cancer is driven by specific pathogenic strains

A
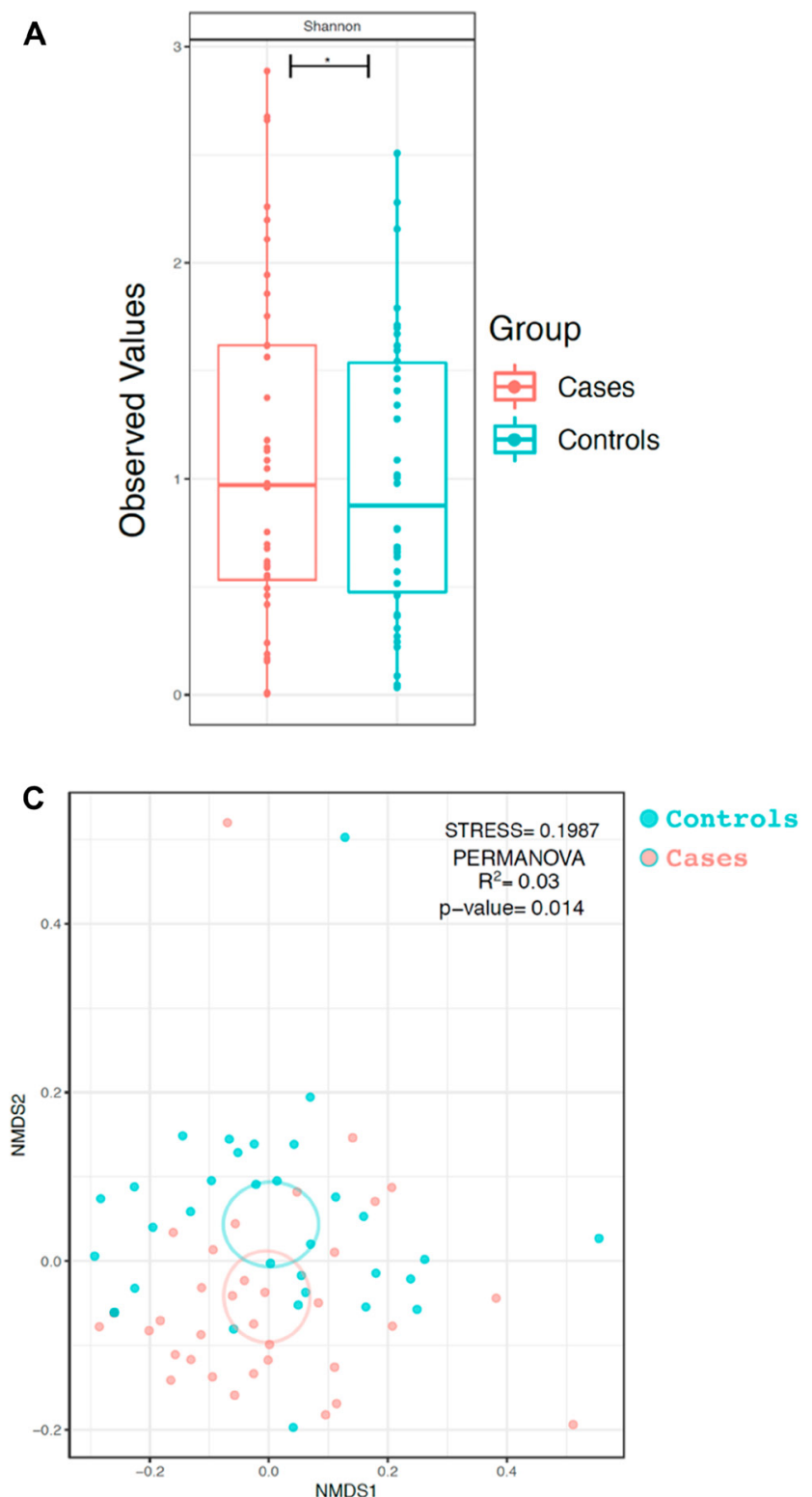

[30-32]. It is, therefore, plausible that our study identified both pathogenic and non-pathogenic $C$. albicans strains. Further research is needed to characterize the specific C. albicans strains associated with HNSCC status. Accomplishing this goal could increase the specificity of a microbiome-based oral wash screening tool for HNSCC. A second fungi of interest, Schizophyllum commune, was enriched in control oral wash. The genera Schizophyllum is a member of the phylum Basidiomycota and has been previously reported as a member of the oral mycobiome [33]. Schizophyllum commune is known to produce the polysaccharide compound schizophylan [34]. Schizophylan has anti-tumor properties in-vitro and showed some promise in treating cancer (including

B
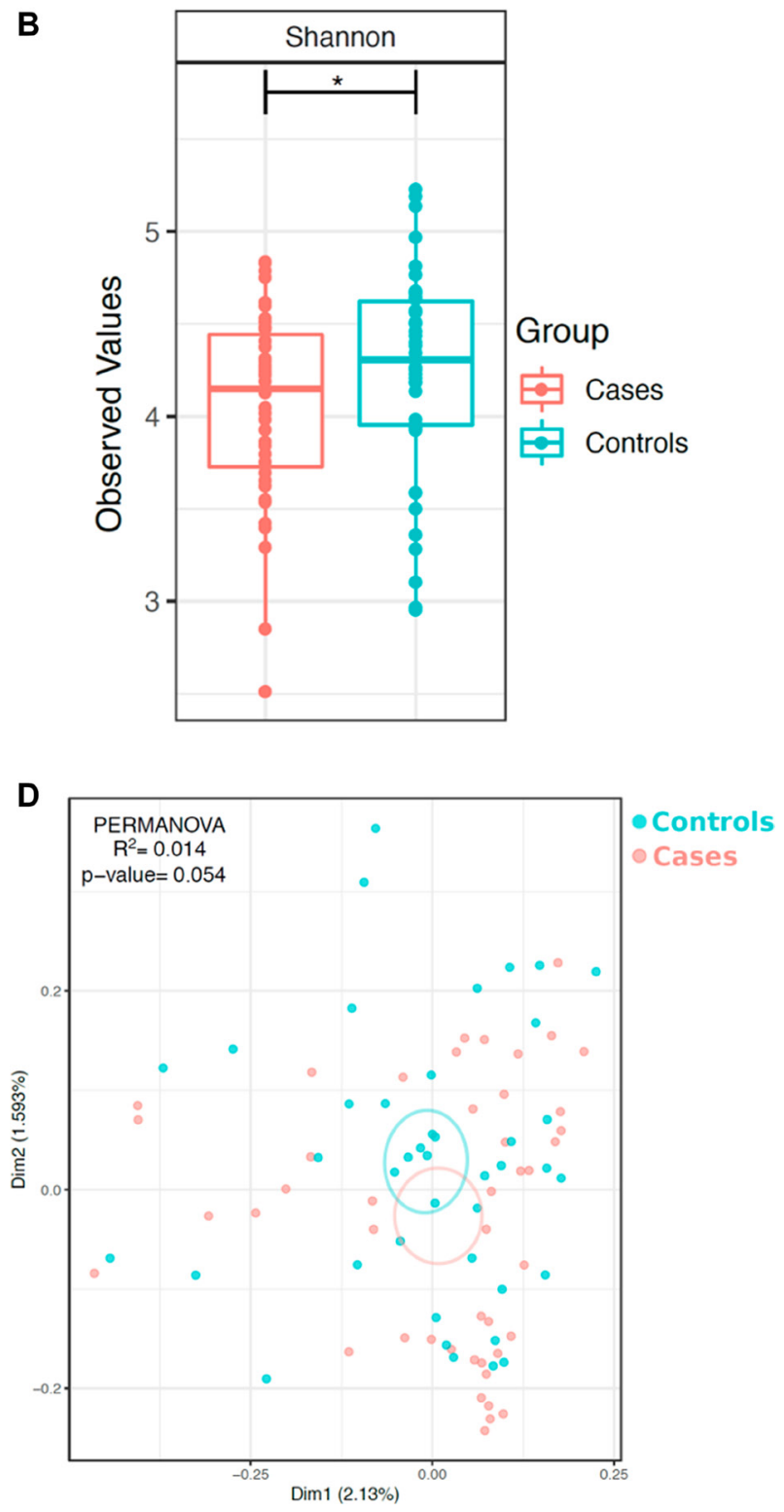

Figure 2: $\alpha$ and $\beta$-diversity of bacterial and fungal communities in HNSCC participant versus control participant oral wash. $\alpha$ diversity of the (A) bacteriome and (B) mycobiome based on cancer status. $\beta$ diversity of the (C) bacteriome and (D) mycobiome based on cancer status. ${ }^{*} p<0.05,{ }^{* *} p<0.01,{ }^{* * *} p<0.001$ 
HNSCC) in studies conducted in Japan in the 1980s [34-37]. The enrichment of this organism in control participants, therefore, supports a role for continued investigation into Schizophylan's anti-cancer properties.

We next explored the bacteriome of the samples. A number of the bacterial organisms observed to be overrepresented in our HNSCC cohort have previously been reported to be associated with HNSCC. Species from the genus Gemella, including Gemella sanguinis, have been found to be increased in oral squamous cell carcinoma tissue [38, 39]. Interestingly, Gemella bacteremia has also been associated with subsequent colorectal carcinoma diagnosis [40]. Streptococcus parasanguinis, although considered part of a healthy oral microbiome, has been reported to be associated with tumor site in oral squamous cell carcinomas [38]. Conversely, Streptococcus cristatus, an oral commensal, was depleted in HNSCC versus control oral wash. Streptococcus cristatus co-aggregates with Fusobacterium nucleatum and Zhang et al. reported that Streptococcus cristatus could attenuate inflammation induced by Fusobacterium nucleatum $[41,42]$. The relative depletion of Streptococcus cristatus in HNSCC participant oral wash versus control wash could, therefore, mean the loss of a commensal that reduces cancer-promoting inflammation. Given the association of Fusobacterium nucleatum with cancer status, it was perhaps surprising that we noted the genera Fusobacterium to be enriched in case oral wash. In fact, other species from the genera Fusobacterium, such as Fusobacterium periodonticum, have, in some instances,

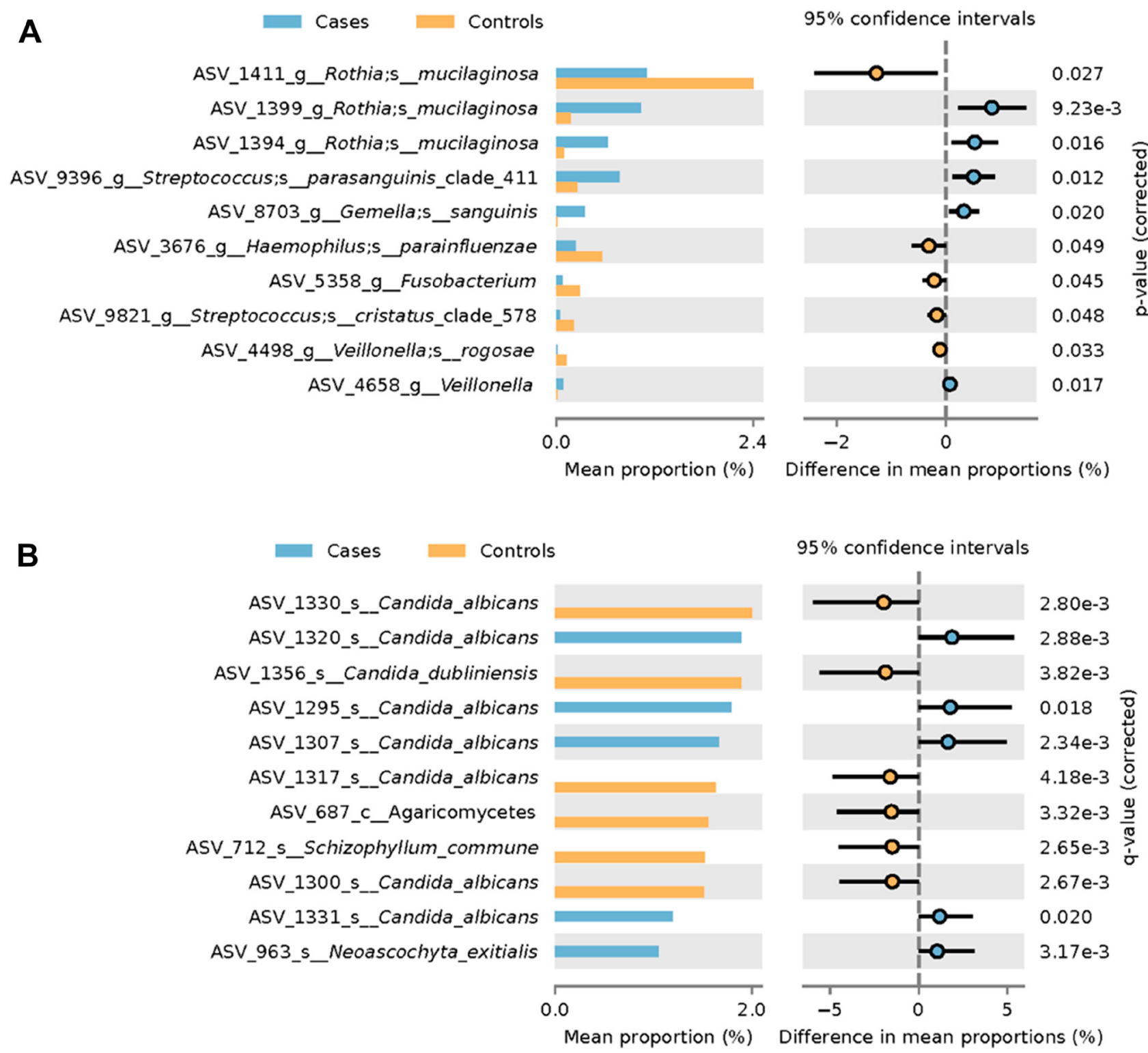

Figure 3: Differential abundance analysis of HNSCC participant versus control participant oral wash. Differential abundance analysis of (A) fungi and (B) bacteria in HNSCC participant versus control participant oral wash. 
been reported to be depleted in HNSCC [43]. Consistent with our results, Rothia has also been reported as both enriched and depleted in HNSCC tissue and biospecimen versus that of control [23, 44, 45].

It is thus apparent that there are distinctions in the relative abundance of bacterial and fungal organisms between the groups. These data provide a basis for a potential screening tool for HNSCC based on bacteriome and mycobiome differences. Interestingly, none of the aforementioned bacterial organisms was found to be associated with an increased risk of HNSCC in a large case-control study nested within two prospective cohort studies that assessed for incident HNSCC risk [46]. It is important to highlight that Hayes et al. profiled prediagnosis oral wash samples; these discrepant findings could reflect differences between the microbiome of individuals predisposed to developing HNSCC and that of individuals with HNSCC pathogenesis-related changes. This type of contrasting observations can help to delineate causation, progression (i. e., pathogenesis) and post-cancer microbiome changes, potentially unrelated to initiation. Large prospective longitudinal studies are needed to further outline the role of bacterial and fungal organisms in the etiology and pathogenesis of HNSCC. Although it

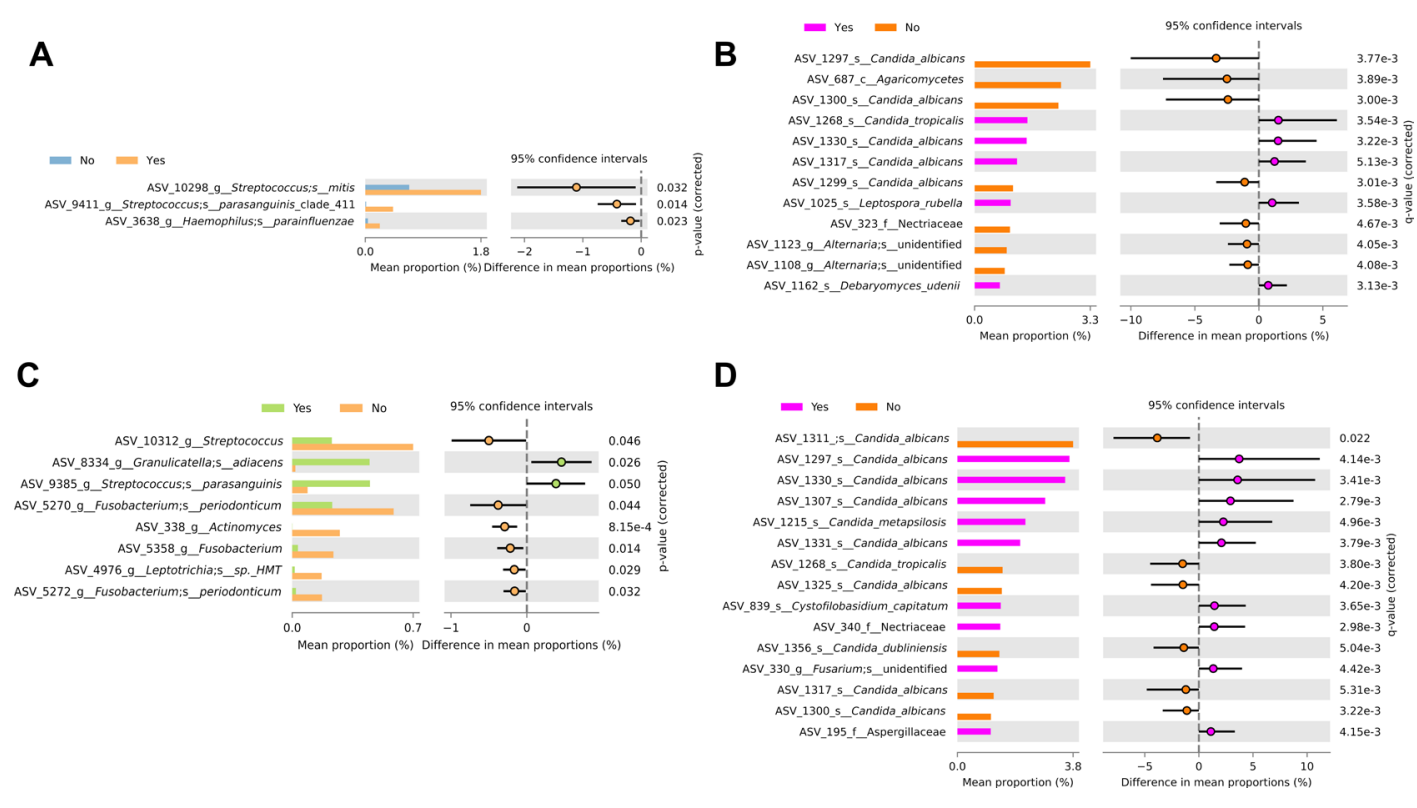

Figure 4: Differential abundance analysis by ethanol use and smoking history. Differential abundance analysis of the (A) bacteriome and (B) mycobiome based on ethanol use. Differential abundance analysis of the (C) bacteriome and (D) mycobiome based on smoking history.
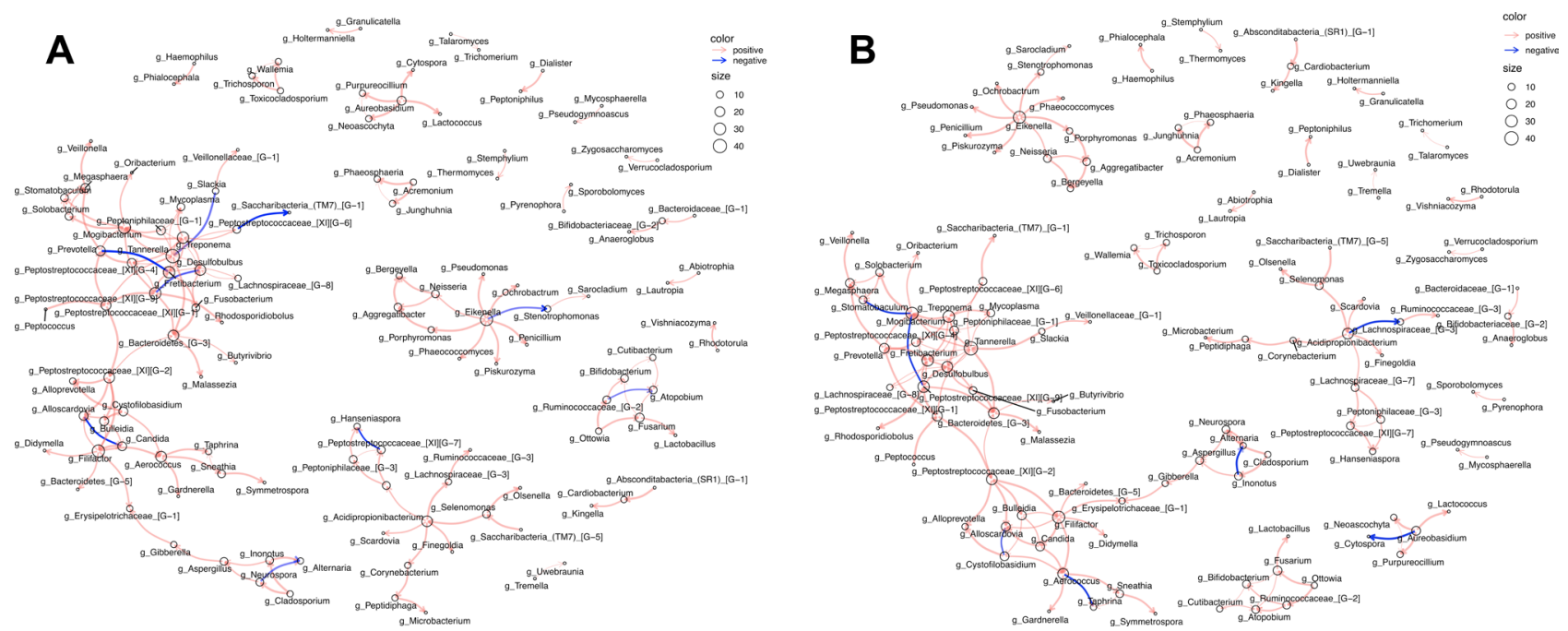

Figure 5: Network analysis depicting intra-kingdom and inter-kingdom correlations. Correlations within (A) case oral wash and (B) control oral wash. 
may be challenging to establish causality, our findings offer an opportunity to further efforts to use oral wash as a screening tool for HNSCC.

The organisms that comprise our microbiome interact with each other. Correlations between organisms present in HNSCC tumor samples and bio specimen have been previously noted. In this study, we found inter and intra-kingdom correlations within oral wash. Although the composition of the clusters within networks appeared largely similar between case and control oral wash, there were some interactions that differed. A positive relationship between two organisms could suggest that they occupy similar niches or even that they share a symbiotic relationship. A negative relationship, by contrast could point to two organisms that either compete against each other through varying means. That we noted multiple interactions that were opposing when considering case oral wash versus control oral wash suggests not only changes in the composition of the microbiome, but also in how members of the microbiome interact with each other in HNSCC patients. The relationship between Alloscardovia and Candida, for example, was negative, in case oral wash but positive in control oral wash. Such shifts could signal the presence of HNSCC in an oral wash based screening tool.

\section{MATERIALS AND METHODS}

\section{Participant enrollment and oral rinse collection}

Approval was obtained from the Institutional Review Board (IRB) for Human Subjects Protection at the Cleveland Clinic. Consent was obtained from patients with HNSCC and normal healthy individuals without malignant dental or airway issues. Participants were asked to provide basic demographic information. Oral rinse was collected prior to surgical treatment, chemotherapy and radiation with the exception of two patients, (one of whom had previously had radiation and surgical treatment, and another who had previously only received radiation). Samples were only collected from participants who had not eaten or drunk anything but water for the previous 30 minutes. Participants were asked to rinse their mouths with normal saline for one minute, which was subsequently spit into a collection cup. This process was repeated once. Oral rinse was processed for storage at the Genomic Medicine Biorepository (GMB) within two hours of collection time. Participants were matched by age, sex and ancestry.

\section{DNA extraction}

Previously extracted and stored DNA was used. The cell pellet was resuspended in 650 ul of lysis buffer, and transferred to TissueLyzer II (Qiagen, Venlo, Netherlands). The TissueLyzer II was set for two rounds of $30 \mathrm{~Hz}$ for 10 minutes. The plates were then centrifuged for 9 minutes at $3000 \mathrm{~g}$ before adding $150 \mathrm{ul}$ of inhibitor removal solution to a new plate along with supernatant from previous plate. The plate was vortexed for 5 seconds, incubated at $4^{\circ} \mathrm{C}$ for 5 minutes and then centrifuged again for 9 minutes at $3000 \mathrm{~g}$. The supernatatant was transferred to a new plate and centrifuged again. The previous step was repeated once. Beads were then added ( $870 \mathrm{ul}$ of a $2 \mathrm{ml}$ bead solution with $85 \mathrm{ml}$ binding solution), the solution mixed and placed on the magnet until solution was clear. The supernatant was discarded and the beads were washed twice using $500 \mathrm{ul}$ of wash buffer. After a final wash step using $500 \mathrm{ul}$ of wash buffer, the supernatant was discarded and $100 \mathrm{ul}$ of water added. The solution was mixed for 10 minutes before placing plate on magnet and transferring $100 \mathrm{ul}$ of extracted DNA for storage.

After undergoing processing using the TissueLyser II (Qiagen), a subset of nineteen samples were instead processed with the Masterpure Yeast DNA purification kit according to manufacturer instructions (Epicentre, Madison, WI, USA). The QIAmp DNA mini kit (Qiagen) was used to complete the DNA purifications. All beads, tubes, and nonenzymatic reagants were treated with UV light for at least 30 minutes prior to use. Reagent controls were confirmed by $16 \mathrm{~S}$ rRNA gene PCR to be absent of contaminating bacteria. Differences in extraction technique were included as a covariate in the analysis.

\section{Amplification and sequencing}

PCR amplification of the V1-V2 region of the 16S rRNA gene and regions of the ITS rRNA gene was accomplished using the QIAseq 16S/ITS Region Panels (Qiagen). PCR was performed with the following conditions for amplification of ITS rRNA gene: 95C for 5 mins, followed by 20 cycles of $95 \mathrm{C}$ for 30 seconds, 50C for 30 seconds, $72 \mathrm{C}$ for $2 \mathrm{mins}$, and an extension of $72 \mathrm{C}$ for $7 \mathrm{mins}$. PCR amplification of the V1-V2 hypervariable regions of the 16S rRNA gene was performed under the following conditions: $95 \mathrm{C}$ for 5 mins, followed by 16 cycles of $95 \mathrm{C}$ for 30 seconds, $50 \mathrm{C}$ for 30 seconds, $72 \mathrm{C}$ for 2 mins, and an extension of $72 \mathrm{C}$ for $7 \mathrm{mins}$. Two rounds of bead purification followed PCR amplification. Adapters were added to the ends of amplicons and libraries were generated using the QIAseq 16S/ITS index kit (Qiagen). The PCR conditions for the index PCR reaction were the same for bacterial and fungal samples and were as follows: 95C for $2 \mathrm{mins}$, followed by 19 cycles of $95 \mathrm{C}$ for 30 seconds, $60 \mathrm{C}$ for 30 seconds, $72 \mathrm{C}$ for $2 \mathrm{mins}$, and an extension of $72 \mathrm{C}$ for $7 \mathrm{mins}$. One round of bead purification of libraries was then performed. Quantification of final libraries was accomplished using the Qubit Fluorometer dsDNA broad range assay. Verification of the appropriate fragment size for each sample was completed with Invitrogen e-Gel. Libraries were pooled in equamolar volumes and the final library pool quantified with qPCR (NEBNext Illumina Library Quant kit). High-throughput sequencing was completed 
at the Case Western Reserve University Genomics Core using the Illumina MiSeq v3 paired-end flow cell after dilution and denaturation of the pool.

\section{Bioinformatic analysis}

The quality of the sequences was assessed using FastQC and MultiQC. Paired end sequences were imported into QIIME 2 (2018.8) using the Casava 1.8 paired-end demultiplexed fastq format [47]. The Divisive Amplicon Denoising Algorithm (DADA) 2 pipeline, within QIIME 2, was used to trim sequences, conduct dereplication, detect and filter chimeric sequences, and merge paired ends [48]. DADA2 uses an algorithm to model and correct amplicon errors and is more reliable than OTU construction methods. The first $20 \mathrm{bp}$ were trimmed from the beginning of bacterial sequences and reads were truncated at $245 \mathrm{bp}$. Fungal sequences were trimmed by $20 \mathrm{bp}$ and were truncated at $250 \mathrm{bp}$. A feature table (the QIIME 2 equivalent of an OTU table), phylogenetic tree and taxonomy file were constructed within QIIME 2. Bacterial sequences were classified against HOMD v 15.1, and fungal sequences against UNITE (Version 18.11.2018) after classifier training within QIIME $2[49,50]$. The output of the dada2 pipeline (feature table of amplicon sequence variants (an ASV table)) was processed for alpha and beta diversity analysis using phyloseq, and microbiomeSeq (http:// www.github.com/umerijaz/microbiomeSeq) packages in $\mathrm{R}$ [51]. Alpha diversity estimates were measured within group categories using estimate_richness function of the phyloseq package [51]. Multidimensional scaling (MDS, also known as principal coordinate analysis; PCoA) was performed using Bray-Curtis dissimilarity matrix between groups and visualized by using ggplot 2 package [52]. We assessed the statistical significance $(P<0.05)$ throughout and whenever necessary, we adjusted $P$-values for multiple comparisons according to the Benjamini and Hochberg method to control False Discovery Rate while performing multiple testing on taxa abundance according to sample categories [53]. We performed an analysis of variance (ANOVA) among sample categories while measuring the of $\alpha$-diversity. Permutational multivariate analysis of variance (PERMANOVA) with 999 permutations was performed on all principal coordinates obtained during PCoA with the ordination function of the microbiomeSeq package. Linear regression (parametric test), and Wilcoxon (Nonparametric) test were performed on ASVs abundances against coprostanol levels using their base functions in $\mathrm{R}$. Co-occurrence patterns were analyzed between features/ taxa (bacterial and fungal taxa) using the "co_occurence network' function in the microbiomeSeq package. The following parameters were used: grouping column = "Variable", rhos $=0.65$, method $=$ "cor", qval threshold $=0.05$. The resulting co-occurrence object was converted into a graph object using igraph 2 and plotted using the ggraph3 package. The size and width of the nodes is proportional to the degree and correlation between the two nodes, respectively. Positive correlations between nodes are represented using the color red and negative correlations are represented using the color blue. The analysis within this study was conducted correcting for age, DNA extraction method, smoking status (tobacco use) and ethanol use.

\section{CONCLUSIONS}

There are distinctions in the oral mycobiome and bacteriome as well as microbiome correlations in the oral cavity, reflected here by oral wash, of HNSCC patients when compared to those of healthy individuals. Corroboration of our findings, particularly in prospective longitudinal studies, could help to further research to facilitate the development of non-invasive strategies to identify high-risk patients based on their oral wash bacteriome and mycobiome profiles. Such studies would help to clarify the temporal order of dysbiosis and carcinogenesis and potentially establish causality. Additionally, we envision the use of probiotics and antifungals to modulate dysbiosis and therefore reduce the risk of HNSCC development and/or pathogenesis. There is already one probiotic product (BIOHM) that combines beneficial bacteria and fungi for improved digestive health [54]. Our research adds to the body of research on the microbiome of HNSCC that would inform the development of a similar product targeted to eliminate HNSCC associated dysbiosis.

\section{Author contributions}

E.S - processing of samples, data analysis, interpretation, writing and revision of manuscript, NS sequencing analysis, data analysis, writing manuscript and critical revision of manuscript, RP- sequencing analysis, critical revision of manuscript, SL- interpretation, critical revision of manuscript, $\mathrm{BB}$ - conception and design of study, procurement of samples, critical revision of manuscript, CE - conception and design of study, data interpretation, writing and critical revision of manuscript.

\section{ACKNOWLEDGMENTS}

The authors would like to thank Lamis Yehia PhD, Jacob Scott MD DPhil, Tammy Sadler MS and Todd Romigh MS for technical advice and critical discussions.

\section{CONFLICTS OF INTEREST}

The authors declare that they have no relevant conflicts of interest. 


\section{FUNDING}

This study was funded, in part, by the VeloSano Program, Cleveland Clinic Taussig Cancer Institute. E.S was a mentee under the auspices of the Doris Duke Clinical Research Mentorship Award (to CE). CE is the Sondra J. and Stephen R. Hardis Endowed Chair in Cancer Genomic Medicine at the Cleveland Clinic, and an ACS Clinical Research Professor.

\section{REFERENCES}

1. Fitzmaurice C, Allen C, Barber RM, Barregard L, Bhutta ZA, Brenner H, Dicker DJ, Chimed-Orchir O, Dandona R, Dandona L, Fleming T, Forouzanfar MH, Hancock J, et al, and Global Burden of Disease Cancer Collaboration. Global, regional, and national cancer incidence, mortality, years of life lost, years lived with disability, and disabilityadjusted life-years for 32 cancer groups, 1990 to 2015: a systematic analysis for the global burden of disease study. JAMA Oncol. 2017; 3:524-48. https://doi.org/10.1001/ jamaoncol.2016.5688. [PubMed]

2. Chaukar DA, Walvekar RR, Das AK, Deshpande MS, Pai PS, Chaturvedi P, Kakade A, D'Cruz AK. Quality of life in head and neck cancer survivors: a cross-sectional survey. Am J Otolaryngol. 2009; 30:176-80. https://doi. org/10.1016/j.amjoto.2008.05.001. [PubMed]

3. Farsi NJ, Rousseau MC, Schlecht N, Castonguay G, Allison P, Nguyen-Tan PF, Souliéres D, Coutlée F, Hier M, Madathil S, Franco EL, Nicolau B. Aetiological heterogeneity of head and neck squamous cell carcinomas: the role of human papillomavirus infections, smoking and alcohol. Carcinogenesis. 2017; 38:1188-95. https://doi. org/10.1093/carcin/bgx106. [PubMed]

4. Blot WJ, McLaughlin JK, Winn DM, Austin DF, Greenberg RS, Preston-Martin S, Bernstein L, Schoenberg JB, Stemhagen A, Fraumeni JF Jr. Smoking and drinking in relation to oral and pharyngeal cancer. Cancer Res. 1988; 48:3282-87. [PubMed]

5. Hashibe M, Brennan P, Chuang SC, Boccia S, Castellsague X, Chen C, Curado MP, Dal Maso L, Daudt AW, Fabianova E, Fernandez L, Wünsch-Filho V, Franceschi $\mathrm{S}$, et al. Interaction between tobacco and alcohol use and the risk of head and neck cancer: pooled analysis in the International Head and Neck Cancer Epidemiology Consortium. Cancer Epidemiol Biomarkers Prev. 2009; 18:541-50. https://doi.org/10.1158/1055-9965.EPI-080347. [PubMed]

6. Shewale JB, Gillison ML. Dynamic factors affecting HPV-attributable fraction for head and neck cancers. Curr Opin Virol. 2019; 39:33-40. https://doi.org/10.1016/j. coviro.2019.07.008. [PubMed]

7. Ndiaye C, Mena M, Alemany L, Arbyn M, Castellsagué X, Laporte L, Bosch FX, de Sanjosé S, Trottier H. HPV DNA, E6/E7 mRNA, and p16INK4a detection in head and neck cancers: a systematic review and meta-analysis. Lancet Oncol. 2014; 15:1319-31. https://doi.org/10.1016/S14702045(14)70471-1. [PubMed]

8. Plummer M, de Martel C, Vignat J, Ferlay J, Bray F, Franceschi S. Global burden of cancers attributable to infections in 2012: a synthetic analysis. Lancet Glob Health. 2016; 4:e609-16. https://doi.org/10.1016/S2214109X(16)30143-7. [PubMed]

9. Madathil SA, Rousseau MC, Wynant W, Schlecht NF, Netuveli G, Franco EL, Nicolau B. Nonlinear association between betel quid chewing and oral cancer: implications for prevention. Oral Oncol. 2016; 60:25-31. https://doi. org/10.1016/j.oraloncology.2016.06.011. [PubMed]

10. Awan KH, Hegde R, Cheever VJ, Carroll W, Khan S, Patil $\mathrm{S}$, Warnakulasuriya $\mathrm{S}$. Oral and pharyngeal cancer risk associated with occupational carcinogenic substances: systematic review. Head Neck. 2018; 40:2724-32. https:// doi.org/10.1002/hed.25486. [PubMed]

11. Gingerich MA, Smith JD, Michmerhuizen NL, Ludwig M, Devenport S, Matovina C, Brenner C, Chinn SB. Comprehensive review of genetic factors contributing to head and neck squamous cell carcinoma development in lowrisk, nontraditional patients. Head Neck. 2018; 40:943-54. https://doi.org/10.1002/hed.25057. [PubMed]

12. Chandrasekharappa SC, Chinn SB, Donovan FX, Chowdhury NI, Kamat A, Adeyemo AA, Thomas JW, Vemulapalli M, Hussey CS, Reid HH, Mullikin JC, Wei Q, Sturgis EM. Assessing the spectrum of germline variation in fanconi anemia genes among patients with head and neck carcinoma before age 50. Cancer. 2017; 123:3943-54. https://doi.org/10.1002/cncr.30802. [PubMed]

13. Kutler DI, Auerbach AD, Satagopan J, Giampietro PF, Batish SD, Huvos AG, Goberdhan A, Shah JP, Singh B. High incidence of head and neck squamous cell carcinoma in patients with fanconi anemia. Arch Otolaryngol Head Neck Surg. 2003; 129:106-12. https://doi.org/10.1001/ archotol.129.1.106. [PubMed]

14. Keeble S, Abel GA, Saunders CL, McPhail S, Walter FM, Neal RD, Rubin GP, Lyratzopoulos G. Variation in promptness of presentation among 10,297 patients subsequently diagnosed with one of 18 cancers: evidence from a national audit of cancer diagnosis in primary care. Int J Cancer. 2014; 135:1220-28. https://doi.org/10.1002/ ijc.28763. [PubMed]

15. Parsonnet J, Friedman GD, Vandersteen DP, Chang Y, Vogelman JH, Orentreich N, Sibley RK. Helicobacter pylori infection and the risk of gastric carcinoma. $\mathrm{N}$ Engl J Med. 1991; 325:1127-31. https://doi.org/10.1056/ NEJM199110173251603. [PubMed]

16. Darveau RP. Periodontitis: a polymicrobial disruption of host homeostasis. Nat Rev Microbiol. 2010; 8:481-90. https://doi.org/10.1038/nrmicro2337. [PubMed]

17. Ahn J, Yang L, Paster BJ, Ganly I, Morris L, Pei Z, Hayes RB. Oral microbiome profiles: 16S rRNA pyrosequencing and microarray assay comparison. PLoS One. 2011; 
6:e22788. https://doi.org/10.1371/journal.pone.0022788. [PubMed]

18. Tezal M, Sullivan MA, Hyland A, Marshall JR, Stoler D, Reid ME, Loree TR, Rigual NR, Merzianu M, Hauck L, Lillis C, Wactawski-Wende J, Scannapieco FA. Chronic periodontitis and the incidence of head and neck squamous cell carcinoma. Cancer Epidemiol Biomarkers Prev. 2009; 18:2406-12. https://doi.org/10.1158/1055-9965.EPI-090334. [PubMed]

19. Ha NH, Park DG, Woo BH, Kim DJ, Choi JI, Park BS, Kim YD, Lee JH, Park HR. Porphyromonas gingivalis increases the invasiveness of oral cancer cells by upregulating IL-8 and MMPs. Cytokine. 2016; 86:64-72. https://doi. org/10.1016/j.cyto.2016.07.013. [PubMed]

20. Bebek G, Bennett KL, Funchain P, Campbell R, Seth R, Scharpf J, Burkey B, Eng C. Microbiomic subprofiles and MDR1 promoter methylation in head and neck squamous cell carcinoma. Hum Mol Genet. 2012; 21:1557-65. https:// doi.org/10.1093/hmg/ddr593. [PubMed]

21. Wang H, Funchain P, Bebek G, Altemus J, Zhang H, Niazi F, Peterson C, Lee WT, Burkey BB, Eng C. Microbiomic differences in tumor and paired-normal tissue in head and neck squamous cell carcinomas. Genome Med. 2017; 9:14. https://doi.org/10.1186/s13073-017-0405-5. [PubMed]

22. Ghannoum MA, Jurevic RJ, Mukherjee PK, Cui F, Sikaroodi M, Naqvi A, Gillevet PM. Characterization of the oral fungal microbiome (mycobiome) in healthy individuals. PLoS Pathog. 2010; 6:e1000713. https://doi. org/10.1371/journal.ppat.1000713. [PubMed]

23. Mukherjee PK, Wang H, Retuerto M, Zhang H, Burkey B, Ghannoum MA, Eng C. Bacteriome and mycobiome associations in oral tongue cancer. Oncotarget. 2017; 8:97273-89. https://doi.org/10.18632/oncotarget.21921. [PubMed]

24. Perera M, Al-Hebshi NN, Perera I, Ipe D, Ulett GC, Speicher DJ, Chen T, Johnson NW. A dysbiotic mycobiome dominated by Candida albicans is identified within oral squamous-cell carcinomas. J Oral Microbiol. 2017; 9:1385369. https://doi.org/10.1080/20002297.2017.13853 69. [PubMed]

25. Vesty A, Gear K, Biswas K, Radcliff FJ, Taylor MW, Douglas RG. Microbial and inflammatory-based salivary biomarkers of head and neck squamous cell carcinoma. Clin Exp Dent Res. 2018; 4:255-62. https://doi.org/10.1002/ cre2.139. [PubMed]

26. Mukherjee PK, Chandra J, Retuerto M, Sikaroodi M, Brown RE, Jurevic R, Salata RA, Lederman MM, Gillevet PM, Ghannoum MA. Oral mycobiome analysis of HIVinfected patients: identification of pichia as an antagonist of opportunistic fungi. PLoS Pathog. 2014; 10:e1003996. https://doi.org/10.1371/journal.ppat.1003996. [PubMed]

27. Zakaria MN, Furuta M, Takeshita T, Shibata Y, Sundari R, Eshima N, Ninomiya T, Yamashita Y. Oral mycobiome in community-dwelling elderly and its relation to oral and general health conditions. Oral Dis. 2017; 23:973-82. https://doi.org/10.1111/odi.12682. [PubMed]

28. Chung LM, Liang JA, Lin CL, Sun LM, Kao CH. Cancer risk in patients with candidiasis: a nationwide populationbased cohort study. Oncotarget. 2017; 8:63562-73. https:// doi.org/10.18632/oncotarget.18855. [PubMed]

29. Chimonidou M, Strati A, Tzitzira A, Sotiropoulou G, Malamos N, Georgoulias V, Lianidou ES. DNA methylation of tumor suppressor and metastasis suppressor genes in circulating tumor cells. Clin Chem. 2011; 57:1169-77. https://doi.org/10.1373/clinchem.2011.165902. [PubMed]

30. Enroth H, Kraaz W, Engstrand L, Nyrén O, Rohan T. Helicobacter pylori strain types and risk of gastric cancer: a case-control study. Cancer Epidemiol Biomarkers Prev. 2000; 9:981-85. [PubMed]

31. Blaser MJ, Perez-Perez GI, Kleanthous H, Cover TL, Peek RM, Chyou PH, Stemmermann GN, Nomura A. Infection with helicobacter pylori strains possessing cagA is associated with an increased risk of developing adenocarcinoma of the stomach. Cancer Res. 1995; 55:2111-15. [PubMed]

32. Loh JT, Shaffer CL, Piazuelo MB, Bravo LE, McClain MS, Correa P, Cover TL. Analysis of cagA in helicobacter pylori strains from Colombian populations with contrasting gastric cancer risk reveals a biomarker for disease severity. Cancer Epidemiol Biomarkers Prev. 2011; 20:2237-49. https://doi.org/10.1158/1055-9965.EPI-110548. [PubMed]

33. Peters BA, Wu J, Hayes RB, Ahn J. The oral fungal mycobiome: characteristics and relation to periodontitis in a pilot study. BMC Microbiol. 2017; 17:157. https://doi. org/10.1186/s12866-017-1064-9. [PubMed]

34. Sung KH, Josewski J, Dübel S, Blankenfeldt W, Rau U. Structural insights into antigen recognition of an anti- $\beta$ $(1,6)-\beta-(1,3)-D-$ glucan antibody. Sci Rep. 2018; 8:13652. https://doi.org/10.1038/s41598-018-31961-x. [PubMed]

35. Kimura Y, Tojima H, Fukase S, Takeda K. Clinical evaluation of sizofilan as assistant immunotherapy in treatment of head and neck cancer. Acta Otolaryngol Suppl. 1994; 511:192-95. https://doi.org/10.3109/0001648940912 8330. [PubMed]

36. Mansour A, Daba A, Baddour N, El-Saadani M, Aleem E. Schizophyllan inhibits the development of mammary and hepatic carcinomas induced by 7,12 dimethylbenz $(\alpha)$ anthracene and decreases cell proliferation: comparison with tamoxifen. J Cancer Res Clin Oncol. 2012; 138:1579-96. https://doi.org/10.1007/s00432-012-1224-0. [PubMed]

37. Okamura K, Suzuki M, Chihara T, Fujiwara A, Fukuda T, Goto S, Ichinohe K, Jimi S, Kasamatsu T, Kawai N. Clinical evaluation of schizophyllan combined with irradiation in patients with cervical cancer. A randomized controlled study. Cancer. 1986; 58:865-72. https:// doi.org/10.1002/1097-0142(19860815)58:4<865::aidcncr2820580411>3.0.co;2-s. [ [PubMed] 
38. Pushalkar S, Ji X, Li Y, Estilo C, Yegnanarayana R, Singh B, Li X, Saxena D. Comparison of oral microbiota in tumor and non-tumor tissues of patients with oral squamous cell carcinoma. BMC Microbiol. 2012; 12:144. https://doi. org/10.1186/1471-2180-12-144. [PubMed]

39. Perera M, Al-Hebshi NN, Speicher DJ, Perera I, Johnson NW. Emerging role of bacteria in oral carcinogenesis: a review with special reference to perio-pathogenic bacteria. J Oral Microbiol. 2016; 8:32762. https://doi.org/10.3402/ jom.v8.32762. [PubMed]

40. Kwong TN, Wang X, Nakatsu G, Chow TC, Tipoe T, Dai RZ, Tsoi KK, Wong MC, Tse G, Chan MT, Chan FK, Ng $\mathrm{SC}, \mathrm{Wu} \mathrm{JC}$, et al. Association between bacteremia from specific microbes and subsequent diagnosis of colorectal cancer. Gastroenterology. 2018; 155:383-390.e8. https:// doi.org/10.1053/j.gastro.2018.04.028. [PubMed]

41. Zhang G, Chen R, Rudney JD. Streptococcus cristatus attenuates fusobacterium nucleatum-induced interleukin-8 expression in oral epithelial cells. J Periodontal Res. 2008; 43:408-16. https://doi.org/10.1111/j.1600-0765.2007.01057.x. [PubMed]

42. Zhang G, Rudney JD. Streptococcus cristatus attenuates fusobacterium nucleatum-induced cytokine expression by influencing pathways converging on nuclear factor- $\mathrm{kB}$. Mol Oral Microbiol. 2011; 26:150-63. https://doi.org/10.1111/ j.2041-1014.2010.00600.x. [PubMed]

43. Guerrero-Preston R, White JR, Godoy-Vitorino F, Rodríguez-Hilario A, Navarro K, González H, Michailidi C, Jedlicka A, Canapp S, Bondy J, Dziedzic A, Mora-Lagos B, Rivera-Alvarez G, et al. High-resolution microbiome profiling uncovers Fusobacterium nucleatum, Lactobacillus gasseri/johnsonii, and Lactobacillus vaginalis associated to oral and oropharyngeal cancer in saliva from HPV positive and HPV negative patients treated with surgery and chemoradiation. Oncotarget. 2017; 8:110931-48. https://doi. org/10.18632/oncotarget.20677. [PubMed]

44. Schmidt BL, Kuczynski J, Bhattacharya A, Huey B, Corby PM, Queiroz EL, Nightingale K, Kerr AR, DeLacure MD, Veeramachaneni R, Olshen AB, Albertson DG. Changes in abundance of oral microbiota associated with oral cancer. PLoS One. 2014; 9:e98741. https://doi.org/10.1371/journal. pone.0098741. [PubMed]

45. Chattopadhyay I, Verma M, Panda M. Role of oral microbiome signatures in diagnosis and prognosis of oral cancer. Technol Cancer Res Treat. 2019;
18:1533033819867354. https://doi.org/10.1177/153303381 9867354. [PubMed]

46. Hayes RB, Ahn J, Fan X, Peters BA, Ma Y, Yang L, Agalliu I, Burk RD, Ganly I, Purdue MP, Freedman ND, Gapstur SM, Pei Z. Association of oral microbiome with risk for incident head and neck squamous cell cancer. JAMA Oncol. 2018; 4:358-65. https://doi.org/10.1001/ jamaoncol.2017.4777. [PubMed]

47. Hall M, Beiko RG. 16S rRNA gene analysis with QIIME2. Methods Mol Biol. 2018; 1849:113-29. https://doi. org/10.1007/978-1-4939-8728-3_8. [PubMed]

48. Callahan BJ, McMurdie PJ, Rosen MJ, Han AW, Johnson AJ, Holmes SP. DADA2: high-resolution sample inference from illumina amplicon data. Nat Methods. 2016; 13:581-83. https://doi.org/10.1038/nmeth.3869. [PubMed]

49. Chen T, Yu WH, Izard J, Baranova OV, Lakshmanan A, Dewhirst FE. The human oral microbiome database: a web accessible resource for investigating oral microbe taxonomic and genomic information. Database (Oxford). 2010; 2010:baq013. https://doi.org/10.1093/database/baq013. [PubMed]

50. Nilsson RH, Larsson KH, Taylor AF, Bengtsson-Palme J, Jeppesen TS, Schigel D, Kennedy P, Picard K, Glöckner FO, Tedersoo L, Saar I, Kõljalg U, Abarenkov K. The UNITE database for molecular identification of fungi: handling dark taxa and parallel taxonomic classifications. Nucleic Acids Res. 2019; 47:D259-64. https://doi.org/10.1093/nar/ gky1022. [PubMed]

51. McMurdie PJ, Holmes S. Phyloseq: an R package for reproducible interactive analysis and graphics of microbiome census data. PLoS One. 2013; 8:e61217. https://doi.org/10.1371/journal.pone.0061217. [PubMed]

52. Wickham H. ggplot2: Elegant Graphics for Data Analysis. New York: Springer-Verlag; 2016.

53. Benjamini Y, Hochberg Y. Controlling the False Discovery Rate: A Practical and Powerful Approach to Multiple Testing. J R Stat Soc Ser B. 1995; 57:289-300. https://doi. org/10.1111/j.2517-6161.1995.tb02031.x.

54. Ghannoum A. BIOHM launches the first probiotic engineered to address the key role of fungus In digestive health. Available 2019 Jun 18, from https://www.forbes. com/sites/afifghannoum/2017/03/07/biohm-launches-the-firstprobiotic-engineered-to-address-the-key-role-of-fungus-indigestive-health/\#186029f0618d. 